\title{
Automatic Negotiation with Mediated Agents in E-commerce Marketplace
}

\author{
Ming-Yu Tsai, Li-Chen Fu, and Ta-Chiun Chou \\ Department of Computer Science and Information Engineering, \\ National Taiwan University, Taipei, Taiwan, R.O.C. \\ \{r91081, lichen, p92001\}@csie.ntu.edu.tw
}

\begin{abstract}
With the prevalence of Internet, many transactions on the traditional market have been successfully replaced by online transactions on the E-commerce market. In this paper, an Agent-Mediated E-mart Framework is proposed. It will be able to accomplish various E-commerce tasks, such as partnership alliance, merchant brokering, and automatic negotiation. Especially, two kinds of automatic negotiations, mutual negotiation and third-party negotiation, are applied to reach an agreement according to user's choice. In addition to the development of this framework, this paper also provides several experiments to show that the outcome of automatic negotiation is satisfactory.
\end{abstract}

\section{Introduction}

Automatic negotiation plays the most important role in the processes of E-commerce. However, there are two critical challenges we need to face here. The first one is to construct a global platform to provide efficient searching, universal publishing, and quick matching. The second challenge is to come up with an autonomous process that can simulate and learn human's behavior to perform commercial activities. This paper proposes an advanced business model, Agent-Mediated E-mart Framework, to meet these challenges.

The rest of this paper is organized as follows. In Section 2, we give the related works. Section 3 proposes the Agent-Mediated E-mart Framework. Section 4 illustrates automatic negotiation. Section 5 demonstrates our experiments. Section 6 concludes this paper.

\section{Related Works}

The major advantage of E-commerce is to bring thousands of fragmented suppliers into a single
E-marketplace and to provide various goods/services to customers from all over the world [1]. There have been several technical reports on investigating E-marketplace with mediated agents and negotiation strategies [2][3][6]. According to each agent's function [5], intelligent agents are categorized into four types - adapter, facilitator, mediator, and broker [4]. The framework of an agent-mediated E-market system was found from [2]. Two relative cooperative and competitive negotiations are presented in [3].

\section{Agent-Mediated E-mart Framework}

\subsection{Overview of Mediated Agents in E-marketplace}

There are four different mediated agents in this framework. Adapter Agent helps buyers/seller to identify and exchange their information. The system arbitrator is named as Coordinator Agent responsible for framework management. Facilitator Agent helps buyers/sellers to establish an alliance. Broker Agent can publish or search goods/services. Negotiator Agent will find the best rewards for both sides.

\subsection{Buyer's Entry Scenario to E-marketplace}

From the buyer's viewpoint, the Adapter Agent will identify the buyer's requirement. Then the Coordinator Agent will play the role of a system coordinator to supervise the entire workflow and to make the agents collaborate more closely. The Broker Agent will search the goods/services from new companies. The Facilitator Agent will find a well-established virtual enterprise to join or construct a new virtual enterprise to wait for other participants. When partnership alliance or merchant brokering stage is completed, the negotiation stage will be executed. If the failed bargain occurs, the Adapter Agent will enter the next round to find another agreement. The detailed flow is shown in Figure 1. 


\subsection{Seller's Entry Scenario to E-marketplace}

On the other hand, from the seller's viewpoint, the Coordinator Agent will complete registrations and authorizations of goods/services. Then, the Adapter Agent publishes goods/services to E-marketplace. The Facilitator Agent will build up the virtual enterprise if partners are available. After partnership alliance or merchant brokering stage is finished, the Negotiator Agent will begin to strike a bargain. If unsuccessful, we will repeat the entire workflow again. The detailed flow is shown in Figure 2.

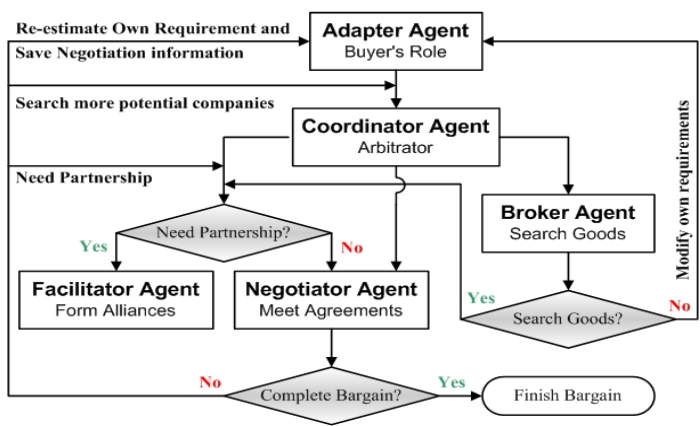

Figure 1. Buyer’s entry scenario in E-marketplace

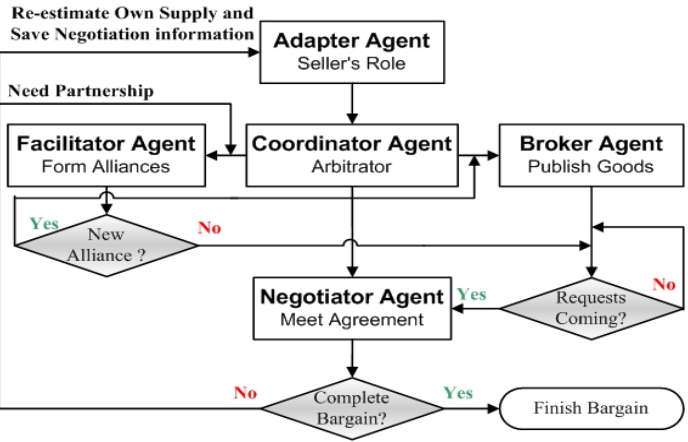

Figure 2. Seller's entry scenario in E-marketplace

\section{Automatic Negotiations}

\subsection{Overview of Automatic Negotiation}

In this paper, two negotiation strategies [3] are described as follows:

- Cooperative Negotiation

For cooperative negotiation, the participants have their own preferences on different trading attributes. Therefore, it is possible for the two parties to obtain satisfactory results after the effective bargain.

- Competitive Negotiation

For competitive negotiation, the objectives of both sides are conflicting. Both sides have the same preferences on the same attributes. Hence, one side losses some benefits when another side gets some.

In the following section, we provide two GA-based negotiation methods, mutual negotiation and third party negotiation, between the two parties.

\subsection{Mutual Negotiation}

In Mutual Negotiation, in order to avoid revealing any private information, both sides do not exchange the fitness function information. But, by adding the opponent's proposal to own GA population, we will estimate opponent's preference and generate the preferable proposal. The flow chart is in Figure 3.

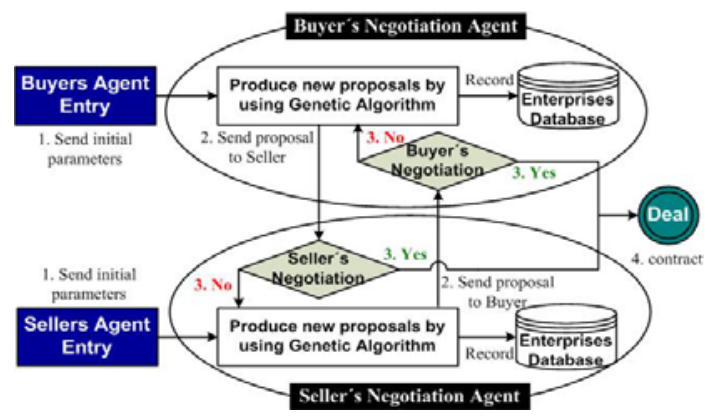

Figure 3. Mutual negotiation

\subsection{Third Party Negotiation}

In Third Party Negotiation, the two parties agree to reveal all preference information to third party. So, through equilibrating two parties' fitness function values, third party will be able to produce the best rewards for both sides. The flow chart is in Figure 4.

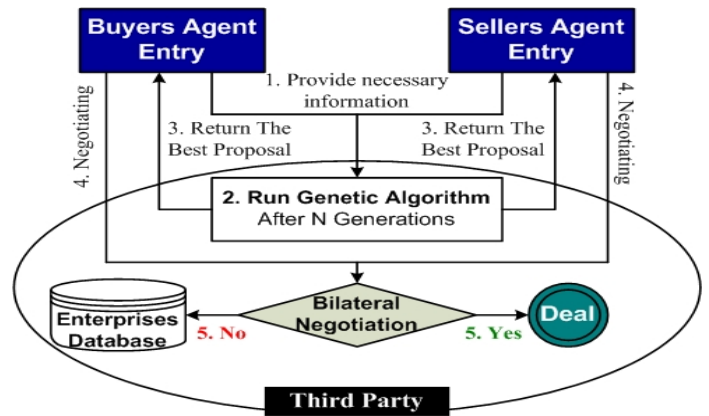

Figure 4. Third party negotiation 


\subsection{Terms of Genetic Algorithm}

As mentioned above, we consider a single product with multiple trading attributes. We have a fitness function:

$$
f_{T A}(t a)=\left\{\begin{array}{c}
\frac{t a}{\| \text { UpLevel }- \text { LowLevel } \|+1}, \text { ta } \in \text { TA } \\
0 \quad \text { ta } \notin T A
\end{array}\right.
$$

In order to combine multiple trading attributes into a fitness function of mutual negotiation, a method named "linear weighted summation" is adopted as shown below:

$F_{\text {Mutual }}(C H)=\sum_{1 \leq j \leq N} W_{j} \cdot f_{T P}\left(t p_{j}\right)$
- $C H:$ Trading proposals of the bargain
- $t p_{j} \in C H:$ Trading proposals
- $N:$ Number of the trading proposals
- $W j:$ Weights of User j.

In the third party negotiation, we use two criteria, Euclidean Distance (ED) and Standard Deviation (SD). The Euclidean distance function is $E D$ (User) = FMutual(User) - Satisfaction(User). If there are $n$ buyers and $m$ sellers in this bargain, $n+m$ users' Euclidean distance and Standard deviation will be calculated. The fitness value of the third party can be expressed as FThird-Party $(C H)=W_{E D} \times E D+W_{S D} \times S D$, where $W_{E D}$ and $W_{S D}$ are the weights of ED and SD, respectively. And $W_{E D}+W_{S D}=1$.

A trading proposal (TP) consists of five attributesquality, quantity, delivery, price, and payment. According to different market relations, a chromosome is built up by different combinations. For example, there are $\mathrm{m}$ proposals in a chromosome for one buyer and m sellers' transaction. See Figure 5.

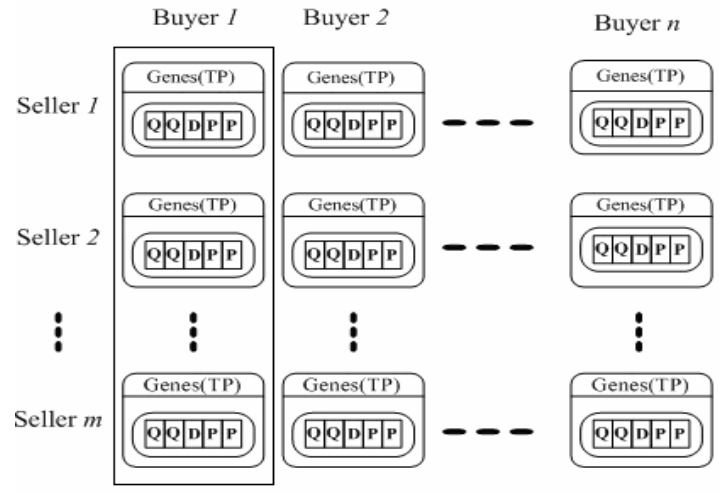

Figure 5. Chromosome representation

\section{Experiment}

In mutual negotiation, we define two terminating conditions. In condition 1, user's fitness value exceeds 0.6 . In condition 2, the number of generation exceeds 1000. In third party negotiation, the negotiation will stop after 1000 generations. Other system parameter setting is described in Table 1.

Table 1. System parameter setting

\begin{tabular}{|c|c|c|c|c|c||}
\hline $\begin{array}{c}\text { Generation } \\
\text { Times }\end{array}$ & $\begin{array}{c}\text { Population } \\
\text { Size }\end{array}$ & $\begin{array}{c}\text { Mutation } \\
\text { Rate }\end{array}$ & $\begin{array}{c}\text { Crossover } \\
\text { Rate }\end{array}$ & $\begin{array}{c}\text { Elitism } \\
\text { Rate }\end{array}$ & $\begin{array}{c}\text { Satisfying } \\
\text { Value }\end{array}$ \\
\hline 1000 & 10 & 0.5 & 0.02 & 0.1 & 0.6 \\
\hline
\end{tabular}

We conduct our experiments based on four different market relations by using random search negotiation, mutual negotiation with condition 1 , mutual negotiation with condition 2 , and third party negotiation.

Fourteen experiment results are shown as Figure 6 $\sim$ Figure 9. Experiment summary is illustrated as the follows. The solutions of random search negotiation are almost the worst results among four negotiation methods. Each GA-based negotiation has its own advantages. Mutual negotiation with condition 1 can produce proposals quickly, but the proposals need to keep on improving. The higher satisfactory fitness value will be created through mutual negotiation with condition 2, but it often sacrifices one side's benefit to get the superior outcome for both sides. Third party negotiation will find better solutions than mutual negotiation.
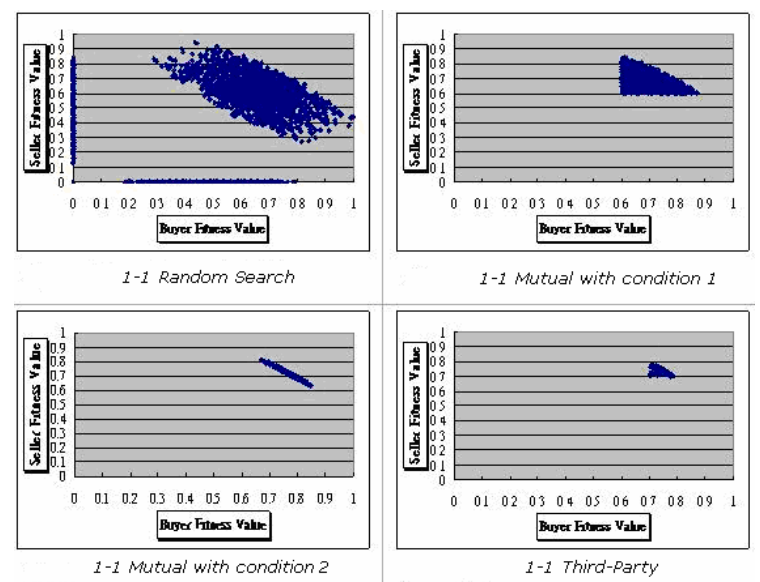

Figure 6. Single buyer vs. single seller 

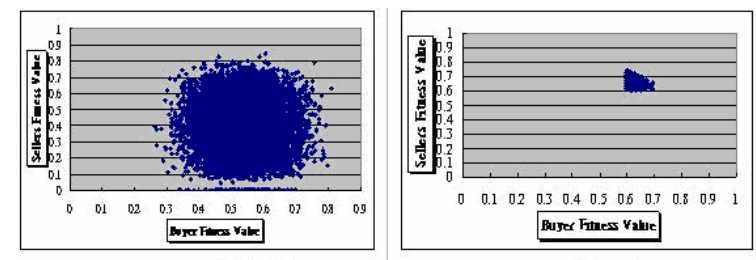

$1-N$ Random Search

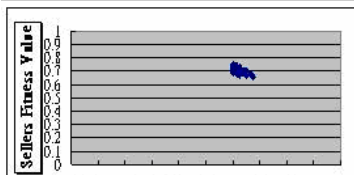

00.10 .203 0.4 0.5 on 0.70 .80 .9 Boyet Finess Yalue

$1-N$ Mutual with condition 2

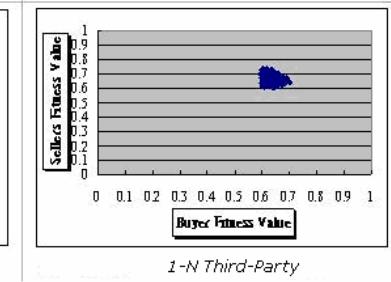

Figure 7. Single buyer vs. multiple sellers
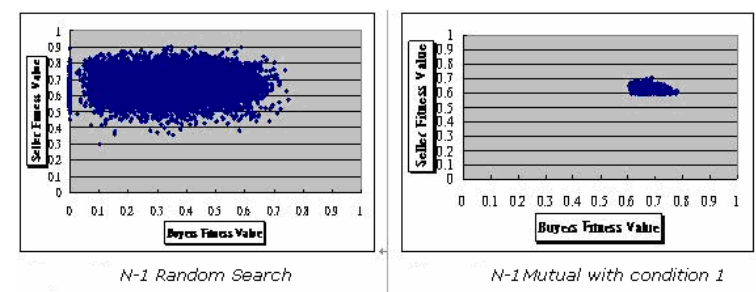

N-1 Random Search

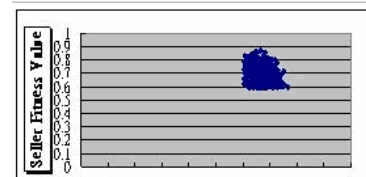

D 0.10 .203 0.4 0.5 on 0.70 .80 .91 Bryers fitues Yalue

$\mathrm{N}-1$ Mutual with condition 2

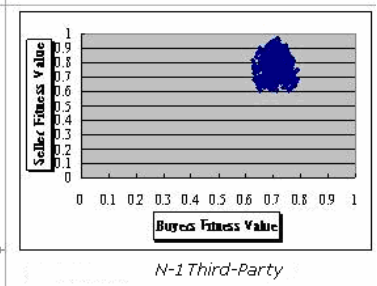

Figure 8. Multiple buyers vs. single seller
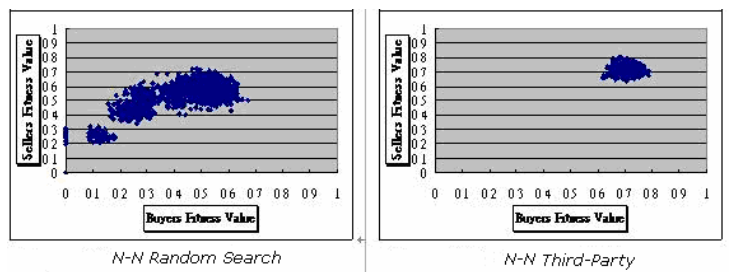

Figure 9. Multiple buyers vs. multiple sellers

\section{Conclusion}

In the second generation of E-commerce, most of the commercial processes would be automatically executed. Therefore, we proposed the Agent-Mediated E-mart Framework. Furthermore, by using the multiple intelligent agents, it will make this framework more spontaneously. Besides, Negotiator Agent will generate a satisfactory outcome for both sides through GA-based algorithm.

In our experiments, two kinds of negotiations called mutual negotiation and third party negotiation are proposed to meet user's requirement. The mutual negotiation has higher privacy to avoid the revelation of own information. According to user's choice, mutual negotiation with condition 1 can find suitable proposals rapidly. However, mutual negotiation with condition 2 will find the proposals with higher fitness function value. On the other hand, third party negotiation can get all of the fitness function information from both sides. Hence, third party can equilibrate each user' fitness function value and find the best benefits for buyers and sellers.

Finally, it is our belief that the best automatic negotiation should be based on transaction environment and user's requirement.

\section{References}

[1] Laudon, K. C. and Traver, C. G., E-commerce: business. technology. society., Addison Wesley, 2002.

[2] Mavetera, N. and Kadyamatimba, A., "A comprehensive agent-mediated e-market framework," in Proc. 5th Int. Conf. on Electronic Commerce, Sep. 2003.

[3] Guttman, R. H. and Maes, P., “Cooperative vs. Competitive Multi-Agent Negotiations in Retail Electronic Commerce", in Proc. Second Int. Workshop on Cooperative Information Agents II, Learning, Mobility and Electronic Commerce for Information Discovery on the Internet, Jul. 1998, pp. 135-147.

[4] Shen, W., "Distributed manufacturing scheduling using intelligent agents,” IEEE Trans. Intelligent Systems, vol. 17, Jan/Feb. 2002, pp. 88-94.

[5] Hu, J. and Grefen, P., "Component based system framework for dynamic B2B interaction,” in Proc. 26th Annual Int. Conf. on Computer Software and Applications, Aug. 2002, pp. 557-562.

[6] Raymund June-ray Lin, Automated Multi-Issue Negotiations between Computational Agents in a Dynamic Environment, PhD thesis, National Taiwan University, Taiwan, 2003. 